\title{
EXTRACTION OF AQUEOUS MINERALS ON MARS SURFACE USING CRISM BASED TARGETED REDUCED DATA RECORDS
}

\author{
R. Gurunadham ${ }^{\text {a }}$, Shashi Kumar ${ }^{\text {b }}$ \\ ${ }^{\text {a }}$ Centre for Remote Sensing, Department of Geo-Engineering, Andhra University - rgurunadh.405@gmail.com \\ ${ }^{\mathrm{b}}$ Photogrammetry \& Remote Sensing department, Indian Institute of Remote Sensing - sksiirs@ gmail.com
}

KEY WORDS: Mars, CRISM, Gale Crater, Phyllosilicates, modified Gaussian model (MGM)

\begin{abstract}
:
Many scientific studies have been carried out to extract the aqueous mineral signatures on the surface of Mars, which has a record of all minerals such as silicates form by magmatic processes and aqueous minerals in the presence of watery environment. To observe these watery conditions, a visible/shortwave infrared mineral mapping camera on Mars Reconnaissance Orbiter (MRO) called Compact Reconnaissance Imaging Spectrometer for Mars (CRISM) is used. The aim of this research is to extract the aqueous minerals on Mars using CRISM sensor. Gale Crater is selected for this study because of its past liquid water history.Gale is $\sim 154 \mathrm{~km}$ in diameter and centered near $5.3^{0} \mathrm{~S}, 138^{0} \mathrm{E}$. Gale Crater has an interior mound named as 'Aeolis Mons', which is nearly $100 \mathrm{~km}$ wide and $5 \mathrm{~km}$ high, consisting of layered sulfates and phyllosilicates. The CRISM reflectance (I/F) targeted reduced data records data of Gale crater, FRT000233AC, centred at $4^{0} 25^{\prime} \mathrm{S}$ and $137^{\circ} 20^{\prime} \mathrm{E}$ with high spatial $(18 \mathrm{~m}, 35 \mathrm{~m} /$ pixel) and spectral resolution (362-1020 nm (VNIR), 1002-3920 nm (IR), $655 \mathrm{~nm} /$ channel) with 545 bands is acquired for this study. The detection and quantification of minerals has been carried out by using a model called modified Gaussian model (MGM). MGM is an approach that uses modified Gaussians in wave number space to model absorption shapes and fits them to a reflectance spectrum.
\end{abstract}

\section{INTRODUCTION}

\subsection{Background}

The curiosity of scientists about Mars is to know does it really had sustainable conditions for the presence of life. To answer this question, there is a need to understand the Mars climatic conditions, water and its atmosphere. Mars is an inner solar system rocky planet and also called as a terrestrial planet \& red planet [1]. Since the 1970's, the Martian atmosphere is formed with both $\mathrm{CO}_{2}$ and $\mathrm{H}_{2} \mathrm{O}$ clouds [2]. Atmospheric dust aerosols and $\mathrm{CO}_{2}$ clouds are affecting the Martian temperature i.e. heating rates of Mars [3].

\section{2 $\mathrm{H}_{2} \mathrm{O}$ in the Martian atmosphere}

The Martian atmosphere is majorly mixed with $\mathrm{CO}_{2}, \mathrm{~N}_{2}, \mathrm{H}_{2} \mathrm{O}$ and other gases with a percentage of $95 \%, 2.7 \%, 0.03 \%$ and the remaining respectively [1]. Highest amount of water is found in northern polar region during northern summer. 'The southern polar region of Mars contains lower amount of $\mathrm{H}_{2} \mathrm{O}$ because of the seasonal $\mathrm{CO}_{2}$ layer and a dust cover inhabiting exchange of water vapour in the atmosphere'. The water content on the Martian surface is low because it has low pressure \& low temperature [4].

\subsection{Gale Crater}

Gale crater is a large Martian impact crater $\sim 154 \mathrm{~km}$ in diameter centred at $5.3^{\circ} \mathrm{S}$ latitude, $222^{\circ} \mathrm{W}$ longitude in the Aeolis Manse region $\left(\sim 10^{\circ} \mathrm{N}-10^{\circ} \mathrm{S}, 240^{\circ} \mathrm{W}-210^{\circ} \mathrm{W}\right)$ [7]. Gale crater lies on north-south dichotomy boundary [17][7], which is the topographic discontinuity that divides the heavily cratered uplands from the smoother and low-elevation northern lowlands [7]. The central mound of Gale crater is named as 'Aeolis Mons' (informally called as Mount Sharp), which is approximately $100 \mathrm{~km}$ wide and 5 kilo meters height and represents the 'thickest exposed stratigraphic reference section for environmental change on Mars' stated by Grotzinger \& Milliken, 2012 [16]. Aeolis Mons is representing the last remaining record of a portion of early Mars history [17]. The reason for choosing the Gale Crater as the study area of this research is that it has the evidence for the presence of phyllosilicates and past liquid water. The scientific studies of the Aeolis Mons reveal that it was filled with a large amount of sediment in the early days [7].

\subsection{Compact Reconnaissance Imaging Spectrometer for Mars}

The Compact Reconnaissance Imaging Spectrometer for Mars (CRISM) is a powerful, technically innovative mineral mapping camera and/or visible/shortwave infrared hyper spectral imager on Mars Reconnaissance Orbiter (MRO) [10]. It is landed by the Mars Science Laboratory rover Curiosity on $5^{\text {th }}$ August, 2005 and is designed to conduct an investigation of modern and ancient habitable environments [15]. The main objective of this sensor is to map Mars atmospheric processes, and characterize past liquid water environments [10]. The basic characteristics of CRISM are shown in table 1.

\begin{tabular}{|l|c|l|l|}
\hline \multicolumn{2}{|l|}{ Spectral Resolution } & $\begin{array}{l}\text { Spatial } \\
\text { Resolution }\end{array}$ & $\begin{array}{l}\text { Swath } \\
\text { Width }\end{array}$ \\
\hline VNIR & IR & $18 \mathrm{~m} /$ pixel & $9.5-12 \mathrm{~km}$ \\
\hline $0.36-1.02 \mu \mathrm{m}$ & $1.02-3.92 \mu \mathrm{m}$ & 12 . \\
\hline \multicolumn{2}{|c|}{ Table 1. Basic characteristics of CRISM }
\end{tabular}

The mineralogy mapping of Mars surface is done with high spatial and spectral resolutions shown in table 1 . This will be done by two detectors called VNIR (for visible and nearinfrared), IR (for short-wave infrared) [9]. CRISM has both multispectral and hyper spectral data sets. CRISM has observing modes as Experimental Data Record (EDR), Derived Data Record (DDR), Limb Data Record (LDR), Targeted Reduced Data Record (TRDR), Calibration Data Records (CDR), Ancillary Data Records (ADR), Map-Projected Multispectral Reduced Data Record (MRDR), and Map- 
Projected Targeted Reduced Data Record (MTRDR) [11]. Targeted reduced data Records (TRDR) is selected for this study as it has a spatial resolution of $18 \mathrm{~m} /$ pixel. TRDR is having high spatial and spectral resolutions as compared to Multispectral Reduced Data Records. Here in this research we are extracting aqueous minerals (Phyllosilicates and $\mathrm{OH}$ ) of Gale Crater using Modified Gaussian Modeling (MGM) for CRISM TRDR data sets and data products. CRISM on MRO provide the evidence of phyllosilicates on the Martian surface. The Ferrous/Magnesium hydrated phyllosilicates and Aluminium hydrated minerals were identified using CRISM sensor stated by A.Brown, J.Bishop. The MRO is the seventh successful orbital mission on Mars. Its primary objectives are to [10]

- Characterize the climate and seasonal variations of Mars according to the water content.

- Search for evidence of past and present liquid water environments.

- Map the sediments, geology and the layered surface deposits.

\section{STUDY AREA}

The data sets of Gale FRT000233AC_07_IF165L_TRR3.IMG, FRT000233AC_07_IF165S_TRR3.IMG are downloaded from the Planetary Data System (PDS) Geosciences node under Mars Orbital data Explorer website sited below as following http://ode.rsl.wustl.edu/mars/index/Datasets.aspx. The CRISM reflectance $(\mathrm{I} / \mathrm{F})$ targeted reduced data records data of Aeolis Mons of Gale crater, FRT000233AC, is centered at $4^{0} 25^{\prime} \mathrm{S}$ and $137^{\circ} 20^{\prime} \mathrm{E}$ with high spatial of $18 \mathrm{~m} /$ pixel and spectral resolution of 0.36-1.05 $\mu \mathrm{m}$ for VNIR data, and 1.02-3.92 $\mu \mathrm{m}$ for IR data with $0.6 \mu \mathrm{m} /$ channel having a total of 545 bands. VNIR data is acquired with 107 bands while IR data is with 435 bands.

\section{METHODS \& MATERIALS}

The reflectance data (I/F) from CRISM is measured simultaneously for up to 545 different wavelengths from 0.36 to $3.92 \mu \mathrm{m}$, giving a spectrum in the visible to near-infrared which allows studies of both the minerals in the surface and the constituents in the Martian atmosphere stated by Murchie et al., 2007. The CRISM TRDR data processing is done by using CRISM Analysis Toolkit (CAT) software. TRDR Hyperspectral data set (FRT000233AC) of Gale Crater is downloaded from PDS geosciences node website. Typical CRISM data processing steps are given below

- Photometric correction

- Atmospheric correction

- $\quad$ CRISM data filtering (CIRRUS)

1. Striping correction (VNIR and IR)

2. Spiking correction (IR)

- Geometric projection of CRISM data

- Calculation of summary parameters

- Modified Gaussian Model

\subsection{Photometric Correction}

The Lambertian (cos $[i]$ ) photometric correction is done by dividing the reflectance (I/F) CRISM image with cosine of incidence angle [cos (i)], which considers the lambertian surface with bidirectional reflectance by resulting a plane surface i.e. lambert albedo [12]. Incidence angle of the used CRISM dataset FRT000233AC is 53.824920654. The Lambertian albedo $\left(\mathrm{A}_{\mathrm{L}}\right)$ with the solar incidence angle (i) is calculated as

$$
\begin{gathered}
\mathrm{A}_{\mathrm{L}}=(\mathrm{I} / \mathrm{F}) / \cos (53.824920654) \\
\mathrm{A}_{\mathrm{L}}=-(\mathrm{I} / \mathrm{F}) / 0.9139653
\end{gathered}
$$

The resultant Lambert albedo $\left(\mathbf{A}_{\mathbf{L}}\right)$ image equals structure of the CRISM reflectance image to a plane surface. [30].

\subsection{Atmospheric Correction}

An important processing step of CRISM data is atmospheric correction, which removes all the $\mathrm{CO}_{2}$ absorption bands by a scaled atmospheric transmission spectrum obtained during an observation crossing Olympus Mons crater. The atmospheric correction is a standard and general function applied to all bands under $3.5 \mathrm{um}$ of the atmospheric transmission. It is derived from the difference of the spectrum measured at the summit of the Olympus Mons volcano (the highest point on the surface of Mars) and the base of it. The results of these corrections are images consisting of absolute reflectance values [13]. Atmospheric correction is required for the spectral imagery before it is used to identify rocks, surface minerals and other materials. A technique called 'Volcano-scan' atmospheric correction is used to remove all $\mathrm{CO} 2$ absorption bands. Many of the CRISM data analysis have been undertaken using Volcanoscan technique [5]. The data are divided by the cosine of the solar incidence angle and by a scaled atmospheric transmission spectrum obtained during an observation crossing Olympus Mons stated by Bibring et al., 2005; Mustard et al., 2008. This corrects for atmospheric gases but not aerosols. The reflectance (I/F) data comes directly from TRDRs, and solar incidence angles from the DDRs [14]. The volcano scan technique removes the absorption bands of $\mathrm{CO} 2$ by ensuring that the Lambert albedo is the same at two wavelengths: $1.890 \mu \mathrm{m}$ and $2.011 \mu \mathrm{m}$ assuming that the first wavelength outside the $\mathrm{CO} 2$ gas bands and the second wavelength deep inside the $\mathrm{CO} 2$ gas bands. The following Volcano-scan model is used for the atmospheric transmission and surface reflection [5].

$$
\begin{gathered}
\mathrm{T}(\lambda)=\text { Transmission }=\frac{\text { Base }}{\text { Summit }} \\
\mathrm{I} / \mathrm{F}(\lambda)=\mathrm{A}_{\mathrm{L}}(\lambda) \cos \left(\theta_{i}\right)(\mathrm{T}(\lambda))^{\beta}
\end{gathered}
$$

Where $I / F(\lambda)$ is reflectance at a wave length $\lambda, A_{L}(\lambda)$ is the Lambertian albedo at the wavelength $\lambda, \theta_{\mathrm{i}}$ is the angle of incidence of sunlight, $T(\lambda)$ is the Olympus Mons reference transmission spectrum, $\beta$ allows correction for relative differences in the surface pressure between base-to-summit of Olympus Mons to other locations.

\subsection{CRISM Data Filtering}

Coolers, Required for CRISM IR data, are less efficient and require occasional power toggling. IR detectors are used only at particular part of time. When the coolers are off only CRISM 
VNIR data is taken. So noise will be part of IR data. The output of Despike will remove that noise. CRISM IR data is affected by the vertical stripes. Stripes are like columns which have DN (Digital Number) s. The affected or missing columns will be recovered by taking average of the adjacent DNs. The output of Destripe will recover those missing columns.

\subsection{Geometric Projection}

A projection is an algorithm or equation for mapping a three dimensional body onto a two dimensional surface such as paper, a computer screen, or in our case, a digital image. For Mars topographic mapping, three conformal map projections called as Mercator, Lambert, and Polar Stereographic are used. The Mercator projection is used for equatorial band, Lambert for medium latitudes, and polar stereographic for the Polar Regions [6]. Polar stereographic projection is used to map the CRISM images as the study area of this research i.e. Gale Crater is located at the polar region.

\subsection{CRISM Summary Products}

Summary parameters are derived to find the region of interest of minerals in the targeted image and these are succeeded in capturing the mineral absorption features at specific wavelengths. The idea of utilizing spectral parameters to analyze spectral data has been used with success in past analysis of spectral data from Mars stated by Bell et al., Murchie et al., 2000. The concept behind these spectral parameters is that a given spectral feature can be captured by a single parameter value, which is calculated by applying an algorithm using combinations of spectral bands to the data [18]. The spectral parameters test the presence of absorption bands and the features of slope or spectral shoulder based on simple arithmetic between spectral channels. The spectral channel (SC) arithmetic equation is stated by Clark and Roush, 1984, and is given as [19]

$$
\mathbf{S C}=\mathbf{1}-\frac{\langle r\rangle_{\lambda \text { band }}}{\langle r\rangle_{\lambda_{\text {continum }}}}
$$

Where $r$ is the reflectance, $\lambda$ band and $\lambda_{\text {continuum }}$ is each a small interval of spectral channels which compute the median value < $\mathrm{r}>$ of the reflectance spectrum. For example R1900 means reflectance at 1900 micron wavelength. The summary parameters used in this study is illustrated with formula is shown in table 2.

\subsection{The Modified Gaussian Model}

The modified Gaussian model (MGM) is 'an approach that uses modified Gaussians in wave number space to model absorption shapes and fits them to a reflectance spectrum using Total Inversion fitting technique' stated by Tarantola and Velette, 1982 [21]. The MGM is a method for deconvolving Spectra as a sum of modified Gaussian distributions superimposed on a baseline continuum that is defined by a slope and an offset and can be linear in wavelength or in wave number (see Fig 1). MGM computations are carried out in energy and natural log reflectance space thus overlapping absorptions are additive and can be modeled using linear inverse theory stated by Sunshine et al., 1990 [20]. The sample MGM fit for the Martian composite spectrum is shown in Fig 1.

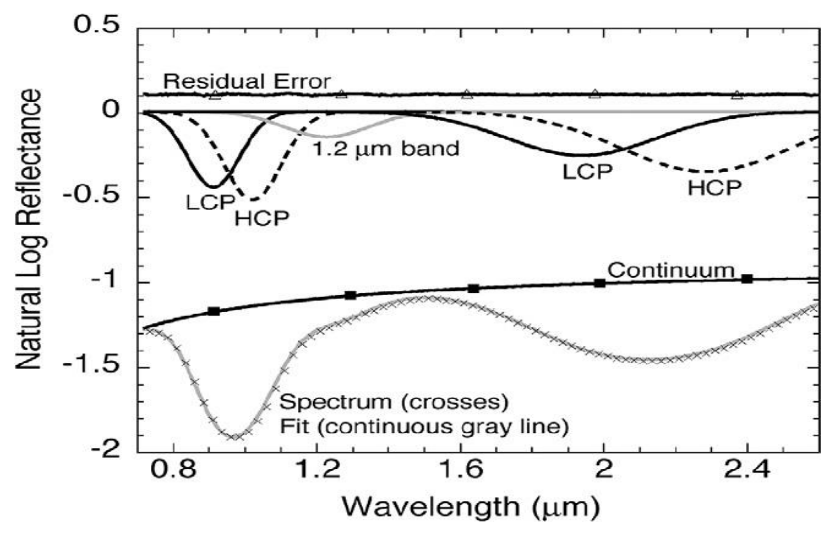

Figure 1. Sample MGM fit for the Martian composite spectrum

The modified Gaussian model is a code that was used in this study for the calculation of band center, band strength and band width. This code is developed by Sunshine et al., 1990 and Sunshine and Pieters, 1998 and it can be downloaded from the website www.planetary.brown.edu/mgm/. The MGM code is applied on the data, which is taken from the Gale crater, in ASCII file. A start-up file and data file are used to begin MGM modeling. "The starting file consists of stopping criteria (a minimum residual error threshold and a minimum change in residual error: typically 0.002 and 1.0E-05 respectively), the number of absorption bands, and initial estimates for all model parameters, including those defining the continuum" stated by Sunshine et al., 1990 [22]. Gaussians are defined as [23]

$$
g(x)=s \cdot \exp \left(\frac{-(x-\mu)^{2}}{2 \sigma^{2}}\right)
$$

And the Modified Gaussian form for better fit is given as [24]

$$
g(x)=s \cdot \exp \left(\frac{-\left(\frac{1}{x}-\frac{1}{\mu}\right)^{2}}{2 \sigma^{2}}\right)
$$

Where $\mathrm{g}(\mathrm{x})$ is absorbance of the feature, $\mathrm{x}$ is its position, $\mathrm{s}$ is band strength, $\sigma$ is band width, and $\mu$ is the band center. The MGM superimposes several Gaussians onto a continuum to

\begin{tabular}{|c|c|c|c|}
\hline Name & Parameter & Formulation & Rationale \\
\hline BD1900 & $\begin{array}{l}1.9 \\
\text { micron } \\
\mathrm{H}_{2} \mathrm{O} \text { band } \\
\text { depth }\end{array}$ & $\begin{array}{l}1- \\
((\mathrm{R} 1930+\mathrm{R} 1985) * \\
0.5) / \\
(\mathrm{a} \text { *R1875+b*R206 } \\
7))\end{array}$ & $\mathrm{H}_{2} \mathrm{O}$ \\
\hline BD2210 & $\begin{array}{l}2.1 \\
\text { micron } \\
\text { band } \\
\text { depth }\end{array}$ & $\begin{array}{l}1- \\
\left((\mathrm{R} 2120+\mathrm{R} 2140)^{*}\right. \\
0.5) / \\
(\mathrm{a} * \mathrm{R} 1930+\mathrm{b} * \mathrm{R} 225 \\
0))\end{array}$ & $\begin{array}{l}\text { Mono } \\
\text { hydrated } \\
\text { minerals }\end{array}$ \\
\hline D2300 & $\begin{array}{l}2.3 \\
\text { micron } \\
\text { drop-off }\end{array}$ & $\begin{array}{l}1- \\
(\mathrm{R} 2290+\mathrm{R} 2330+\mathrm{R} \\
2350) / \\
(\mathrm{R} 2140+\mathrm{R} 2170+\mathrm{R} \\
2210)\end{array}$ & $\begin{array}{l}\text { Hydrated } \\
\text { mineral will } \\
\text { be }>1\end{array}$ \\
\hline
\end{tabular}
model the spectrum with known absorption features at fixed wavelengths due to specific electronics transmissions [23].

Table 2. CRISM surface composition summary parameters 


\section{RESULTS \& DISCUSSIONS}

The $\mathrm{CO}_{2}$ dominated Martian atmosphere has strong $\mathrm{CO}_{2}$ absorptions between 1.4 and $2.2 \mu \mathrm{m}$ regions. The Carbon dioxide features between $1.555-1.665 \mu \mathrm{m}$ and at $1.45 \& 2 \mu \mathrm{m}$ are corrected by using the volcano scan atmospheric correction. The absorptions of aqueous minerals are masked with $\mathrm{CO}_{2}$ gas at 1.9 to $2.1 \mu \mathrm{m}$ wavelengths. These masked absorptions have been removed using this atmospheric correction.

\subsection{Histogram Based Segmentation of Summary parameters}

The map projected CRISM data is given as the input for the calculation of summary parameters. 'For most of the band depths and similar measures of absorptions, Zero is the minimum realistic value. The $99^{\text {th }}$ percentile is a good maximum for better results. The "minimum maximum" values vary from one parameter to another parameter' stated by $K$. Seelos et al., 2012 [2]. The histogram based segmentation has been applied to CRISM summary parameters i.e. D2300, BD2210, BD1900 by using the threshold values and those threshold values are shown in table 3 . The $2300 \mathrm{~nm}$ drop off, $2210 \mathrm{~nm}$ band depth and $1900 \mathrm{~nm}$ band depth summary parameters, D2300, BD2210 and BD1900 are applied for the CRISM image FRT000233AC taken from the Gale Crater. As a result these summary parameters applied images are showing the absorption depths having negative values, which are occurred possibly at the boundaries of the crater.

\begin{tabular}{|c|c|c|}
\hline Parameter & $\begin{array}{l}\text { Minimum } \\
\text { (distribution peak) } \\
\text { stretch }\end{array}$ & $\begin{array}{l}\text { Maximum }\left(99^{\text {th }}\right. \\
\text { percentile }) \text { Stretch }\end{array}$ \\
\hline $\mathrm{D} 2300$ & 0.001 & 0.025 \\
\hline BD2210 & 0.003 & 0.020 \\
\hline BD1900 & 0.001 & 0.042 \\
\hline
\end{tabular}

Table 3. Threshold values used for D2300, BD2210 \& BD1900

\subsection{CRISM mineral mapping}

The aim of this research is to extract the phyllosilicate minerals from the Gale Crater. For this observation, RGB color combination of Phyllosilicate mineralogy is used for the summary parameters i.e. D2300, BD2210, and BD1900. Red channel is selected with D2300, Green channel is selected with BD2210, and Blue channel is selected with BD1900 and these combinations would be observed from the table 5. RGB color composite according to the table 5 is taken and the resultant mineral mapping of CRISM image is shown in Fig 4. A color combination image of Phyllosilicate mineral parameters i.e. D2300, BD2210, and BD1900 (in the red, green and blue channels respectively) for CRISM image FRT000233AC displays $\mathrm{Fe} / \mathrm{Mg}$ phyllosilicates.

\begin{tabular}{|l|l|l|l|}
\hline Channel & Parameter & $\begin{array}{l}\text { Minimum } \\
\text { (distribution } \\
\text { peak) } \\
\text { stretch }\end{array}$ & $\begin{array}{l}\text { Maximum } \\
\left(99^{\text {th }}\right. \\
\text { percentile) } \\
\text { Stretch }\end{array}$ \\
\hline Red & D2300 & 0.003 & 0.028 \\
\hline Green & BD2210 & 0.001 & 0.020 \\
\hline Blue & BD1900 & 0.014 & 0.046 \\
\hline
\end{tabular}

Table 4. Threshold values used for Histogram based segmentation of RGB composite
Red colored pixels in the Fig 4 represent $\mathrm{Mg} / \mathrm{Fe}$-clay mineral absorption features, green pixels indicate Aluminium-clay absorption features. RGB color stretches for the parameters D2300, BD2210, and BD1900 between 'minimum maximum' thresholds i.e. $0.003,0.001$, and 0.014 (distribution peak) to $0.028,0.020$, and 0.046 respectively are shown in table 4 .

\begin{tabular}{|c|c|c|}
\hline Channel & $\begin{array}{l}\text { CRISM } \\
\text { Summary } \\
\text { Parameter }\end{array}$ & $\begin{array}{l}\text { Histogram } \\
\text { Scaling }\end{array}$ \\
\hline Red & D2300 & $\begin{array}{c}0.005-0.02 \\
\text { to 0-250 }\end{array}$ \\
\hline Green & BD2210 & $\begin{array}{c}0.005-0.02 \\
\text { to 0-250 }\end{array}$ \\
\hline Blue & BD1900 & $\begin{array}{c}0.01-0.04 \\
\text { to 0-250 }\end{array}$ \\
\hline
\end{tabular}

Table 5. RGB color composite for Phyllosilicate (PHY) mineralogy

Band parameters D2300, BD2210 and BD1900 are selected to highlight the presence of iron/magnesium phyllosilicates and to build a RGB colored CRISM image (see Fig 4). D2300 parameter shows the $\mathrm{Fe} / \mathrm{Mg}$ phyllosilicate absorption features (approximately at $2.3 \mu \mathrm{m}$ ).

\subsection{ROI-based endmember extraction}

The spectral signature of interest of the given D2300 summary parameter is to get the endmember spectra of the band threshold D2300. From the Fig 2(a), the red pixel marks are due to $2.3 \mu \mathrm{m}$ drop off, D2300, and 368 pixels are given as the threshold for the CRISM reflectance image in the range of 0.0124 to 0.02 . The $2.3 \mu \mathrm{m}$ absorption bands are considered as the region of interest (ROI) pixels to identify particular mineral. Some of the pixels in the image are taken as ROI, 450 pixels, and those pixels (red colored pixels) are masked with green colored polygon. The green color masked pixels are shown in the Fig 2 (b), considered as Numerator for ratio spectra. The Numerator mean spectrum is assumed as $\mathrm{S} 1$ and it is shown in the Fig 3. The dusty or unremarkable pixels or flat pixels from the same image are masked with blue color polygon shown in Fig 2(b), considered as Denominator for ratio spectra. The Denominator mean spectrum is assumed as S2 and it is shown in Fig 3.

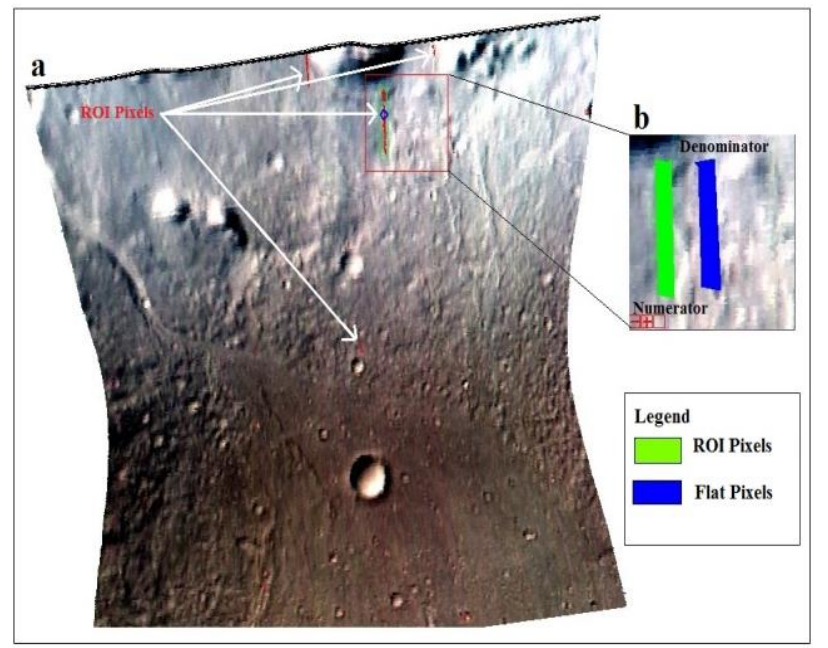

Figure 2. ROI-based selection of CRISM IR image 
CRISM ratio spectra is calculated by taking the numerator spectrum (S1) is divided with the denominator spectrum (S2).The resultant ratio spectra (endmember spectra) is marked in red color and is shown in Fig 3. The continuum removed ratio spectra $(\mathrm{S} 1 / \mathrm{S} 2)$ is compared with the CRISM phyllosilicate spectral library using Spectral Feature Fitting (SFF) model to best possible mineral estimation. Out of all the Phyllosilicate mineral types, only Illite Lail04 is matched with the ratio spectra $(\mathrm{S} 1 / \mathrm{S} 2)$ taken from the image FRT000233AC.

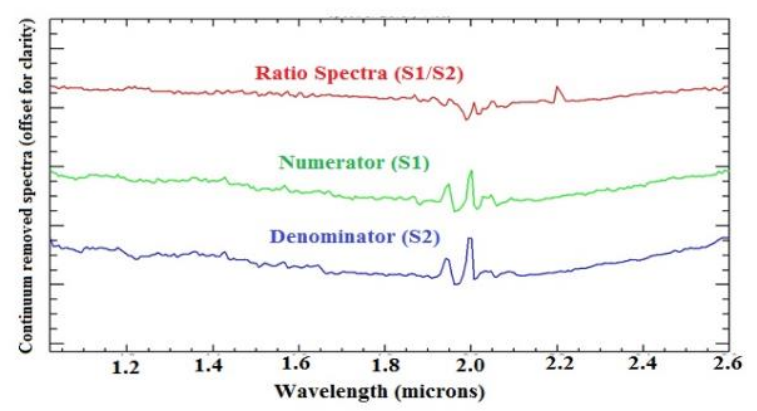

Figure 3. CRISM ratio endmember spectra

4.3.1 Illite $\left(\left\{\left(\mathrm{K}, \mathrm{H}_{3} \mathrm{O}\right)(\mathrm{Al}, \mathrm{Mg}, \mathrm{Fe})_{2}(\mathrm{Si}, \mathrm{Al})_{4} \mathrm{O}_{10}\left[(\mathrm{OH})_{2}\right.\right.\right.$, $\left.\left.\left.\left(\mathbf{H}_{2} \mathbf{O}\right)\right]\right\}\right)$ : Illite is a non-expanding, clay-sized mineral. Illite is a Phyllosilicate or layered alumino-silicate. It comes under the category of Mica-Phyllosilicates. The absorption depths of the illite sample are measured for that three absorption features. The absorption features are centred at $1.41 \mu \mathrm{m}, 1.95$ and $2.20 \mu \mathrm{m}$ and the band depths of the band vary from $4.4 \%$ to $27.1 \%$. The combination of $\mathrm{H}-\mathrm{O}-\mathrm{H}, \mathrm{OH}$, and metal $-\mathrm{OH}$ bonds produce prominent absorption features at 1.0 to $2.40 \mu \mathrm{m}$ wavelengths due to the containment of water molecules, hydroxyls, and $\mathrm{Al}$, $\mathrm{Fe}$, and $\mathrm{Mg}$ ions in the minerals [6].

\subsection{Modified Gaussian Model}

The Modified Gaussian model (MGM) is applied on CRISM reflectance data which was collected from the central mound of the Gale Crater. The wavelength (nanometers) and reflectance values taken from the CRISM rationed spectra (from both VNIR and IR data) are given as the input to this MGM to run the model. The wavelength range is selected from $400 \mathrm{~nm}$ to $2600 \mathrm{~nm}$ and the natural log reflectance range is selected from 2 to 0.5 to fit the CRISM model spectra. After applying the MGM to the spectra, initial absorption band parameters (Center, Full Width Half Maximum, and Strength) and Root Mean Square (RMS) error are obtained. Initially there is more uncertainty in the fit due more residual error present in the spectra. The residual error is nothing but the difference between the spectrum and the fit. More number of iterations is performed until the fit gets minimum residual error. Total of 170 iterations are carried out to get the complete fit of the spectra. After the completion of 170 iterations the residual error gets minimized i.e., initially the residual error is 0.9 and it is reduced to 0.001 at which the complete fit is obtained. The final absorption band parameters are obtained representing the shift in the band absorption features compared to the initial MGM fit of the CRISM spectra. The initial and final MGM absorption band parameters are shown in table 6 and table 7 respectively.

\begin{tabular}{|c|c|c|c|}
\hline S. No & Center & $\begin{array}{c}\text { Full Width Half } \\
\text { Maximum (FWHM) }\end{array}$ & Strength \\
\hline 1 & 333.000 & 400.0 & -2.0000 \\
\hline 2 & 1000.000 & 250.0 & -1.0000 \\
\hline 3 & 2000.000 & 300.0 & -1.0000 \\
\hline
\end{tabular}

Table 6. Initial absorption band parameters (Center, FWHM, and Strength) of the MGM fit

\begin{tabular}{|c|c|c|c|}
\hline $\begin{array}{c}\text { S. } \\
\text { No }\end{array}$ & Center & $\begin{array}{c}\text { Full Width Half } \\
\text { Maximum (FWHM) }\end{array}$ & Strength \\
\hline 1 & 333.896 & 126.5138 & -0.1669 \\
\hline 2 & 898.147 & 143.1176 & -0.6497 \\
\hline 3 & 1845.743 & 428.0376 & -1.3507 \\
\hline
\end{tabular}

Table 7. Final absorption band parameters (Center, FWHM, and Strength) of the MGM fit

The final MGM fit has the absorptions same as the CRISM ratio spectra i.e. the absorptions at $1.4,1.9$ and $2.2 \mu \mathrm{m}$ wavelength. These absorptions representing the Magnesium and Ferrous phyllosilicates are matched with the ratio spectra in this study. Due to the presence of $\mathrm{Mg} / \mathrm{Fe}$ clay in the Gale crater, there is a chance that the illite is formed in watery conditions.

\section{CONCLUSIONS}

This word section gives the conclusions of the research work carried out. All the process is carried out only for CRISM IR data except in MGM process as the CRISM VNIR data has a spectral range of 0.39 to $1.05 \mu \mathrm{m}$. The newly developed volcano scan atmospheric correction technique is very useful to remove the $\mathrm{CO}_{2}$ absorptions present at 2 micron region in the CRISM Targeted Reduced Data Record (TRDR) data taken from the Gale crater. New version TRR3 for CRISM TRDR dataset gave good result for the correction of $\mathrm{CO}_{2}$ absorptions at 2-micron region. According to the $\mathrm{CO}_{2}$ atmosphere present in the Olympus Mons crater only, the volcano scan atmospheric correction removes the $\mathrm{CO}_{2}$ present in our data. As the Martian atmosphere is full of dust aerosols; the volcano scan algorithm is concentrated only to remove $\mathrm{CO}_{2}$ absorptions and treated as there are no aerosols. CRISM Full resolution Targeted (FRT) images have a spatial resolution of $18 \mathrm{~m} / \mathrm{pixel}$, which is very useful in the distribution of important mineral classes and finding the small aqueous mineral signatures on the Martian surface. Summary parameters played an important role in finding the particular mineral absorption features. By using the D2300, BD2210 and BD1900 summary parameters, the location of the Phyllosilicate minerals in the image are easily identified. These summary parameters are the most representative of the surface but these are not unique for a specific mineral, this is one of the disadvantages of using summary products. MGM model developed by Sunshine et al., 1990 is a compositional analysis tool to reduce the residual error present in the CRISM spectra and to better estimation of mineral without having spectral library. The band center, band strength and band width of the CRISM data are acquired as the initial and final band parameters using the modified Gaussian model and these parameters have proved to direct detection of mineral from the data given. From all the obtained results it is clear that the presence of aqueous minerals (phyllosilicates) in the Aeolis 
Mons of Gale Crater on Mars. The presence of phyllosilicates on Gale Crater represents that water flowed on that surface for some period of time.

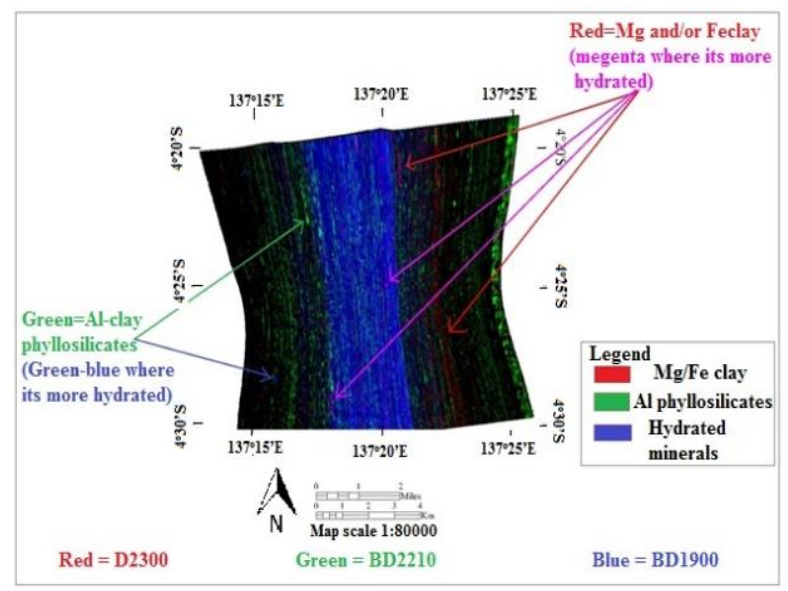

Figure 4. Mineral mapping of CRISM observation FRT000233AC

\section{REFERENCES}

[1] S.V.S Murty, 2012, Scientific Aspects of Exploration of Mars, PLANEX, Physical Research Laboratory Ahmedabad, 114.

[2] J. Hurley, N.A. Teanby, P.G.J. Irwin, S.B. Calcutt, 2014, Differentiability and retrievability of $\mathrm{CO}_{2}$ and $\mathrm{H}_{2} \mathrm{O}$ clouds on Mars from MRO/MCS measurements: A radiative-transfer study, Planetary and space science, Vol 97, 65-84.

[3]Mark T. Lemmon, Michael J. Wolff, James F. Bell III, 2014, Dust aerosol, clouds, and the atmospheric optical depth record over 5 Mars years of the Mars Exploration Rover mission, Icarus, 1-16.

[4]Mads Dam EllehØj, 2007, subsurface ice on Mars: A study on ground ice stability and applications, NIELS BOHR INSTITUTE, 1-158.

[5]P. C. McGuire et al., 2009, an improvement to the volcanoscan algorithm for atmospheric correction of CRISM and OMEGA spectral data, Planetary and Space Science 57, 809815.

[6]Chaojun Fan et al., 2012, Analysis of United states geological survey spectral library of silicate minerals: implication for remote sensing applications, Journal of Applied Remote sensing, 1-11

[7] B. J. Thomson, N. T. Bridges, R. Milliken, 2011, Constraints on the origin and evolution of the layered mound in Gale Crater, Mars using Mars Reconnaissance Orbiter data, Icarus, Vol 214, 413-432.

[8]C. E. Viviano, F. P. Seelos, S. 1. Murchie et al., 2014, revised CRISM spectral parameters and summary products, Lunar and Planetary Science Conference, 2444-2445.

[9]Xavier Ceamanos, 2010, Spectral smile correction of CRISM/MRO hyperspectral images, IEEE Transactions on Geosciences and Remote Sensing, 48, 3951-3959.

[10]Scott L. Murchie, 2006, Investigating Martian History with the CRISM Imaging Spectrometer, Johns Hopkins APL Technical Digist, 27,145-155.

[11]Scott Murchie, 2012, CRISM data product software interface specification version 1.3.7.1, 28-32.
[12]F. Seelos, S. Murchie, 2012, MRO CRISM map projected targeted reduced data records (MTRDRs): Data processing pipeline and product set, CRISM data user's workshop, 1-67. [13]Mohit Melwani Daswani, 2011, Mineral Spectra Extraction and Analysis of the Surface Mineralogy of Mars with Hyperspectral Remote Sensing, 1-62.

[14]Scott L. Murchie, Frank P. seelos, 2009, Compact Reconnaissance Imaging Spectrometer for Mars investigation and data set from Mars Reconnaissance Orbiter's primary science phase, Journal of Geophysical Research, Vol. 114, 1-15 [15] A. R. Vasavada, D. F. Blake, J. Crisp et. al., 2013, Mars science laboratory: First 100 sols monitoring the atmosphere and environment within Gale Crater, Lunar and Planetary Science Conference, 1191-1192.

[16]James J. Wray, 2011, Gale Crater: The Mars Science Laboratory/Curiosity Rover landing Site, School of Earth and Atmospheric Sciences, 1-45.

[17] K. S. Edgett, 2010, Curiosity's Candidate Field Site in Gale Crater, Mars, $4^{\text {th }}$ MSL Landing site workshop, 1-45.

[18]Pelkey et al., 2007, CRISM multispectral summary products: parameterizing mineral diversity on Mars from reflectance, Journal of Geophysical Research, Vol. 112, 1-18.

[19]J. Carter, F. Poulet, S. Murchie et al., 2013, automated processing of planetary hyperspectral datasets for the extraction of weal mineral signatures and applications to CRISM observations of hydrated silicates on Mars, Planetary and Space Science, Vol. 76, 53-67.

[20]Lisa C. Kanner, John F. Mustard et al., 2007, assessing the limits of the Modified Gaussian Model for remote spectroscopic studies of pyroxenes on Mars, ICARUS, vol. 187, 442-456.

[21]Mario Parente, Heather D. Makarewicz, Janice L. Bishop, 2011, Decomposition of mineral absorption bands using nonlinear least squares curve fitting: Application to Martian meteorites and CRISM data, Planetary and Space Science, vol.59, 423-442.

[22]J. M. Sunshine, C. M. Pieters et al., 1999, Availability of Modified Gaussian Model, Brown University, 1-2.

[23] John R. Skok, John F. Mustard, Scott L. Murchie et al., 2010, spectrally distinct ejecta in Syrtis Major, Mars: Evidence for environmental change at the Hesperian-Amazonian boundary, Journal of geophysical research, Vol. 115, 1-19.

[24] E. A. Gallie, D. A. Lyder, B. Rivard \& E. A. Cloutis, 2008, Technical Note: Equivalence of modified Gaussian model (MGM) in wave number and Gaussian in wavelength for deconvolution of hyperspectral reflectance spectra, International journal of Remote sensing, 29:14, 4089-4096. 